Mots. Les langages du politique

$86 \mid 2008$

Toponymes. Instruments et enjeux

\title{
Le nom de pays comme outil de représentation sociale
}

Georgeta Cislaru

\section{OpenEdition}

Journals

Édition électronique

URL : https://journals.openedition.org/mots/13452

DOI : $10.4000 /$ mots. 13452

ISSN : 1960-6001

Éditeur

ENS Éditions

Édition imprimée

Date de publication : 30 mars 2008

Pagination : 53-64

ISBN : 978-2-84788-129-5

ISSN : 0243-6450

Référence électronique

Georgeta Cislaru, "Le nom de pays comme outil de représentation sociale », Mots. Les langages du politique [En ligne], 86 | 2008, mis en ligne le 30 mars 2010, consulté le 23 avril 2022. URL : http:// journals.openedition.org/mots/13452 ; DOI : https://doi.org/10.4000/mots.13452

(c) ENS Éditions 


\section{Le nom de pays comme outil de représentation sociale ${ }^{\star}$}

Noms de lieux, toponymes, mais aussi toponymes politiques ou politonymes... Dans le cas des noms de pays, la géographie semble n'être qu'un prétexte identitaire: toponymes bien sûr, mais surtout politonymes dans leur usage médiatique, les noms de pays ont une force de représentation symbolique transcendantale. En effet, le nom de pays ne se limite pas à désigner le territoire national : il en configure et en rassemble le contenu à travers des emplois polyréférentiels: «L'Irak vote aujourd'hui », "Les États-Unis ont bombardé l'Afghanistan », "La France a battu l’Italie 2 à 1 », «L'Ukraine ferme ses frontières», etc. II rend ainsi saillants différents représentants de la nation - armée, équipes nationales, gouvernement - tout en résumant temporairement l'essence de la nation à l'un de ses représentants. Par ailleurs, en prenant en charge tantôt la référence à l'état, tantôt la référence au peuple ou à la nation, le nom de pays sémiotise des contenus symboliques variés.

Ce type d'emploi des noms de pays est tellement fréquent dans la presse que Michelle Lecolle l'assimile à une routine journalistique (Lecolle, 2001a et $2001 b)$. Mais, s’il est vrai que ces emplois, considérés généralement comme métonymiques (et donc, tropiques), ne génèrent pas d'effet rhétorique, ils jouent en revanche un rôle social indéniable. D'une part, les emplois polyréférentiels ont un effet pragmatique marqué, dû au fait que les noms de pays prennent en charge référentiellement les différents acteurs des évènements mis en scène. D'autre part, la fréquence de ces emplois associe durablement les noms de pays aux concepts d'État et de nation.

Sorte d'intégrateurs sémantiques, les noms de pays assurent ainsi une représentation unitaire de l'état-nation (Cislaru, 2005). Cela semble légitimer des formules ${ }^{1}$ telles que au nom de la France et je suis/nous sommes

* Nous tenons à remercier les relecteurs, qui nous ont incitée à préciser certains éléments de cet article

1. L'usage que nous faisons du terme formule est proche de la définition donnée par Alice Krieg-Planque: «un objet descriptible dans les catégories de la langue, et dont les pratiques langagières et l'état des rapports d'opinion et de pouvoir à un moment donné au sein

Université Paris 3 (SYLED), gcislaru@univ-paris3.fr 
la France, qui fonctionnent comme des marqueurs linguistiques de la délégation de parole et de l'alchimie de la représentation (voir infra) : la première présuppose - et cautionne? - le statut de porte-parole, la deuxième pousse l'alchimie de la représentation à son point extrême, l'identification effective. Il nous semble que l'usage de ces formules pourrait donner des indications quant à l'implication du toponyme dans la construction des représentations sociales. Nous nous proposons d'étudier ici le fonctionnement discursif des deux formules dans un corpus de presse constitué à partir des archives Factiva. Ces archives contiennent les bases des agences de presse (AFP, Presse canadienne, Reuters, etc.) et une centaine de périodiques francophones, dont les principaux quotidiens et hebdomadaires français ${ }^{2}$. Les options de recherche de la base de données nous ont permis de recenser l'ensemble des occurrences des deux formules et de visualiser leurs contextes grâce à l'affichage du texte de l'article. Les premières occurrences attestées remontent à 1994; nous avons recueilli les dernières données le 14 novembre 2006 .

\section{Des formes linguistiques aux objets sociaux}

Tout d'abord, signalons qu'il existe un lien très fort entre formes linguistiques et formes sociales. En effet, le nom de pays renvoie régulièrement à des concepts sociopolitiques, l'état et la nation étant les plus représentatifs. Ces concepts sociopolitiques sont considérés comme sui-référentiels (Searle, 1998) ou a-référentiels (Kaufmann, 2006), dans le sens où ils renvoient à leur propre définition, n'ayant pas de référent immédiatement et intégralement perceptible dans le monde: les objets sociaux n'existent que par l'accord des hommes, du fait d'une intentionnalité collective scellée par des conventions linguistiques et diffusée par la chaîne communicationnelle. Dès lors, la référenciation s'opère par ce que Laurence Kaufmann appelle - en s'inspirant de Hilary Putnam (1990) - la « déférence sémantique », qui est à la fois une forme de partenariat social consolidant l'intentionnalité collective et un acte d'allégeance tacite à l'usage collectif. Ainsi, les membres d'une communauté s'engagent à «valider à crédit les entités sociales et politiques qui les entourent en endossant le point de vue impersonnel et anonyme de leur groupe d'appartenance», point de vue qui est néanmoins fixé par les «élites politiques et médiatiques $»^{3}$ (Kaufmann, 2006, p. 112, 113).

de l'espace public déterminent le destin [...] à l'intérieur des discours» (Krieg-Planque, 2003, p. 14). Mais le traitement que nous faisons de l'objet se limite exclusivement à sa circulation, en omettant sa genèse (trop éloignée dans notre cas) et donc tout un pan de sa dimension polémique.

2. La Croix, Les Échos, L'Express, Le Figaro, L'Humanité, Libération, Le Monde, Le Point, Sud Ouest, La Tribune, etc.

3. On peut ne pas adhérer à cette conception idéologique élitiste (voir aussi Moscovici, 1984, p. 15-16) pour la simple raison que si le domaine des «masses» et celui des élites étaient 
L'usage du nom de pays en tant qu'intégrateur sémantique assure la mise en place d'une activité référentielle collective, qui donne du «crédit ontologique » (Kaufmann, 2006) aux objets sociaux tels l'État et la nation : à force de récurrences discursives, les emplois polyréférentiels cimentent la réalité des objets sociaux, et la dimension spatiale convoquée par le toponyme assure à ces derniers une matérialité irréfutable. Les représentations sociales que le nom de pays met en place ont par ailleurs un pouvoir explicatif, dans le sens où elles configurent des objets en les rendant accessibles et pertinents pour tout un chacun 4 .

C'est donc en tant qu'outil linguistique de construction de la réalité sociale et de son ordre institutionnel - dans l'optique de John Searle (1998) ainsi que de Peter Berger et Thomas Luckmann (1996) - que nous allons appréhender le nom de pays. Nom de pays et, par délégation, nom d'État / nation, avec tout le pouvoir symbolique qui s'ensuit ${ }^{5}$. La porte est ouverte à une alchimie de la représentation telle qu'elle a été définie par Pierre Bourdieu6, mais s'opérant en deux temps et mettant en avant la dimension dénominative : le groupe endosse le nom de pays; le nom de pays prend en charge le représentant du groupe (porte-parole ou acteur, homme d'État ou institution).

Situé à la charnière du groupe et du porte-parole, le nom de pays occupe une position privilégiée pour rendre compte des implications et dynamiques socio-politiques telles qu'elles peuvent être retracées par l'usage linguistique. Car, comme le souligne Marie-Anne Paveau, «[doté de] la propriété de collectivité, [le nom propre] signifie pour un groupe et ce sens est négocié dans le rapport entre les sujets et leur expérience du monde » (Paveau, 2006, p. 171). Il s'agit donc d'observer les enjeux socio-politiques tels qu'ils se manifestent dans l'investissement symbolique du nom de pays à travers les processus de négociation qui accompagnent son usage. C'est l'usage de la formule au nom de la France, en tant que marqueur de l'alchimie de la représentation, que nous allons étudier dans un premier temps.

\section{L'effet d'oracle : une question de prise en charge}

Grâce à l'effet d'oracle, «le porte-parole fait parler le groupe au nom duquel il parle, parlant ainsi avec toute l'autorité de cet absent insaisissable»

étanches, la transmission et la reprise de ce qui serait fixé par les «élites politiques et médiatiques» serait impossible.

4. «Representations, on the other hand, restore collective awareness and give it shape, explaining objects and events so that they become accessible to everyone and coincide with our immediate interest. » (Moscovici, 1984, p. 22)

5. D'après Émile Durkheim (1958, p. 433) : «l'état, c'est proprement l'ensemble des corps sociaux qui ont seuls qualité pour parler et pour agir au nom de la société.»

6. «Groupe fait homme, [le représentant] personnifie une personne fictive, qu'il arrache à l'état de simple agrégat d'individus séparés, lui permettant d'agir et de parler à travers lui “comme un seul homme”.» (Bourdieu, 1982, p. 101). 
(Bourdieu, 2001, p. 269). En faisant parler ceux au nom de qui on est autorisé à parler, on leur fait prendre en charge ce qui est dit.

On parle et on agit, le plus souvent, au nom de la France. Le nom de pays l'emporte de loin sur le nom de peuple dans la formule légitimatrice, avec 1007 occurrences de au nom de la France contre 39 de au nom des Français dans la presse française (janvier 1994 à novembre 2006). Par ailleurs, si la formule au nom des Français peut être employée par les hommes politiques dans à peu près les mêmes contextes que au nom de la France ${ }^{7}$, elle connaît aussi des emplois non canoniques tels que «au nom de tous les Français, y compris des clients de Canal+ », "au nom des Français qui ne veulent pas de l'élargissement à la Turquie», etc.

Cet écart de distribution entre au nom de la France et au nom des Français indique que ce ne sont pas directement le peuple ou la nation qui autorisent la substitution symbolique, mais la médiation par le territoire qu'opère le nom de pays. La dimension spatiale constitue d'ailleurs le point d'ancrage et de stabilité de l'identité collective française (Cislaru, 2006a et 2006b) en conditionnant une convergence entre état et territoire. Ainsi, la France, nom de lieu et nom d'état, trace les frontières dans lesquelles la nation se reconnaît et se cristallise. De ce fait, l'alchimie de la représentation concerne ici non plus directement le "groupe fait homme» mais l'investissement spatio-étatique, qui cautionne l'identification entre le toponyme et le nom d'une personnalité politique :

Tableau 1. La prise en charge référentielle

\begin{tabular}{|l|l|}
\hline $\begin{array}{l}\text { La France demande l'interdiction des } \\
\text { farines animales en Europe (titre) }\end{array}$ & $\begin{array}{l}\text { Poulets - Bernard Kouchner demande } \\
\text { l'interdiction des farines animales (titre) }\end{array}$ \\
\hline
\end{tabular}

Au nom de la France, Bernard Kouchner, le secrétaire d’État à la Santé, a demandé, hier à Luxembourg, l'interdiction totale de l'utilisation de toutes les farines carnées dans l'alimentation animale en Europe.

\begin{tabular}{l|l} 
(La Tribune, 9 juin 1999) & (Les Échos, g juin 1999)
\end{tabular}

Bernard Kouchner identifié à la France: raccourci référentiel ou, au contraire, médiation de l'alchimie de la représentation? La vicariance du toponyme donne une dimension particulière à l'autorisation symbolique du porteparole: le choix d'utiliser le nom de pays dans le titre renforce le poids de

7. Les deux formules n'en ont pas pour autant le même sens, ni le même pouvoir de légitimation auprès de ceux au nom de qui l'on parle. En témoigne cette remarque d'un lecteur s'adressant à une personnalité politique: «Parlez pour vous, bien à l'abri, sous les ors et les lambris de la République, mais, de grâce, ne vous arrogez pas le droit de parler au nom des Français !» (Le Progrès, 17 avril 2006) 
l'acte, aussi bien sur le plan externe, dans le contexte européen et international, que sur le plan interne, en préconisant une adhésion nationale. La prise en charge est ainsi un acte d'institutionnalisation sociale.

\section{Rituels, légitimation et production idéologique}

Si l'on regarde les champs d'application de la formule au nom de la France, on constate que bon nombre de ses contextes d'emploi révèlent des rituels qui servent à maintenir une certaine représentation de l'appareil de l'État en conférant à ce dernier une validité performative. Mais, comme c'est le nom de pays qui est effectivement employé dans la formule, la validité performative est immédiatement récupérée par ce dernier. Une chaine de déférence sémantique se met en place.

Au nom de la France, on énonce des suggestions et des propositions (notamment dans les institutions européennes), on prend des engagements, on demande des amendements, on promeut des initiatives communautaires, on présente des mémorandums, on signe des protocoles, des accords, des traités, on participe à ou on préside des réunions ou des conseils (au niveau international). Le discours met en scène des évènements qui resteront dans les éphémérides - tel ce 28 janvier 1944 où «Charles de Gaulle, au nom de la France libre, appelle à un nouveau régime avec les colonies d'Afrique " (" éphémérides », La Presse canadienne, 27 janvier 2001), ou ce 2 septembre 1945 où «le général de Lattre de Tassigny a signé au nom de la France l'acte de reddition du Japon sur le port du cuirassé Missouri » (Le Monde, 18 mai 2006) -, ou tout simplement dans le quotidien de l'activité politique, comme c'est le cas de «M. Mattéi, qui s'exprimait au nom de la France, organisatrice de la rencontre » (Agence France Presse, 2 mai 2006), ou encore de «traités antérieurs, tous signés au nom de la France par des gouvernements ou sous un chef d'État socialistes, de Guy Mollet à Lionel Jospin en passant par François Mitterrand » (Le Monde, 26 novembre 2004), etc.

L'action au nom de la France peut aussi s'inscrire dans la durée, comme le suggère l'imparfait dans "Je connais les convictions européennes de Laurent Fabius et ce qu'ont été ses choix lorsqu'il exerçait des responsabilités importantes au nom de la France» (François Hollande dans Sud-Ouest, 27 août 2004). C'est d'ailleurs par ce biais duratif que la formule assoit sa légitimité, en définissant un statut (" être au service de la France»).

Toujours au nom de la France, on agit par la parole: on félicite, on remercie, on reçoit et on présente des condoléances, on rend hommage, on salue (un peuple, son courage, sa dignité, etc.), on présente des vœux ou des félicitations: «dans la matinée, il [Jacques Chirac] avait rendu un "hommage solennel, au nom de la France, à toutes les victimes de la catastrophe dans 
l'océan Indien" " (Le Monde, 5 janvier 2005). Tous ces actes de langage rendent compte de rituels diplomatiques dotés d'une forte charge symbolique.

Historiquement, la formule au nom de la France a permis de prendre possession. Ainsi, "Jacques Cartier prend possession du Canada, au nom de la France; au nom de la France, il [Pierre Savorgnan de Brazza, commissaire général du gouvernement du Congo français] organise la colonie» (AP French Worldstream, 2 octobre 2006). Associée à la formule, la prise de possession transformait les territoires étrangers en territoires français. La référence même du toponyme se voit alors modifiée, avec l'émergence de la France métropolitaine et de la France d'outre-mer.

La formule au nom de la France permet aussi de prendre position, d'annoncer des partis pris idéologiques. Au nom de la France, on défend un principe / une cause / une idée, on mène un combat, on plaide pour. De même, c'est au nom de la France qu'on condamne, qu'on se repent, qu'on pardonne ou qu'on demande pardon. En condamnant les crimes contre l'humanité, en exprimant ses regrets et ses excuses aux accusés d'Outreau, on configure ce qui doit correspondre à l'idéologie de l'état représenté. La continuité de l'état et la continuité de l'idéologie semblent garanties par l'emploi du nom de pays. C'est ainsi que, en s'appuyant sur la continuité et l'unité préconisées par le toponyme, François Mitterrand a pu refuser de faire des excuses au nom de la France pour les crimes commis pendant la guerre et sous le gouvernement de Vichy'. Cependant, une remise en cause de l'unité et de la continuité reste toujours possible étant donné que le toponyme prend en charge avant tout la dimension étatique et que le groupe au nom duquel on parle peut se dissocier de celle-ci ${ }^{10}$.

\section{Quand les hommes politiques parlent au nom de la France}

Les chefs d'État font partie de cette élite politique qui, traditionnellement, est habilitée à conférer aux objets sociaux une naturalité et un pouvoir causal (voir Kaufman, 2006, p. 90-92), et donc à mettre en place et / ou à maintenir des chaînes de reconnaissance et de transmission d'informations sémantiques qui donnent substance aux objets sociaux. Il y a ainsi double délégation:

8. Ces contextes d'emploi de l'expression au nom de la France sont attestés dans plusieurs périodiques des archives Factiva.

9. "Si les mots ont un sens, reconnaître que le "gouvernement de Vichy" a agi et décidé au nom de la France, c'est dire que de Gaulle était un général rebelle et ses compagnons, Français libres et résistants, des aventuriers. C'est dire que la condamnation du général pour trahison était “légitime”. Comment peut-on prétendre que l'état de Vichy incarnait la République?» (Le Monde, 22 juillet 1995).

10. "Quant à Mitterrand, il avait cru devoir lancer: “Je ne ferai pas d'excuses au nom de la France. La République n'a rien à voir avec ça !” C'était à l'automne 1994, au nom de l'état. Aujourd'hui c’est la Nation qui attend le jugement de Maurice Papon. » (La Tribune, 2006 octobre 97) 
celle de la représentation en tant que porte-parole et celle de l'expertise référentielle visant à identifier la substance des objets sociaux. La façon dont les chefs d'état emploient une formule comme au nom de la France est donc doublement significative.

L'emploi qu'en faisait Charles de Gaulle est resté mythique : «Moi, général de Gaulle, soldat et chef français, j'ai conscience de parler au nom de la France » (voir Siblot, 1986). Emploi qui aurait pu ne pas être légitime au départ, étant donné la situation politique de l'époque, mais qui est associé à la libération de la France et à l'héroïsme du général dans l'imaginaire politique ${ }^{11}$. De Gaulle a continué de recourir à la formule pour appuyer ses actes, en lui conférant ainsi une valeur quasi performative, comme dans cette allocution suite au putsch d'Alger:

Au nom de la France, j'ordonne que tous les moyens, je dis tous les moyens, soient employés partout pour barrer la route à ces hommes-là, en attendant de les réduire : j'interdis à tout Français et d'abord à tout soldat d'exécuter aucun de leurs ordres. (Discours du 23 avril 1961)

Comme on a pu le voir ci-dessus, François Mitterrand s’interrogeait sur le sens des mots, lui qui n'avait pas «besoin d'une idée de la France » car il la « vivait ». Il ne parlait pas beaucoup au nom de la France; la formule s'en trouve ainsi sacralisée.

Jacques Chirac, quant à lui, parle soit «au nom de la France» tout court soit «au nom de la France et en son propre nom » ${ }^{12}$ : «En ce jour cruel pour votre peuple, je vous adresse, en mon nom personnel et au nom de la France, mes condoléances attristées et vous assure de la solidarité dans l'épreuve» (AFP, 30 septembre 2006). Si, d'après l'analyse de Pierre Bourdieu, une telle formulation indiquerait que la personne individuelle ne s'annule pas au profit de la personne morale transcendante - or, il faut «mourir» pour devenir institution -, on remarquera que Jacques Chirac rajoute « en mon nom propre» dans des contextes particuliers: lors des rituels diplomatiques qui sous-entendent une politique et une politesse calquées sur les rituels d'interaction (Goffman, 1974).

11. Cette façon d'utiliser la formule permet à Régis Debray de définir l'héroïsme de Charles de Gaulle: «Personne n'atteint au sublime sans frôler le burlesque. De Gaulle était zinzin: il entendait des voix, parlait aux morts, et de lui-même à la troisième personne. Un grand homme est un fantaisiste grave, assez tenace pour désarmer les rires. L'épopée héroï-comique de la France libre commence par des drôleries, et lorsque René Cassin, avant de signer la première convention juridique unissant la Grande-Bretagne aux trois tondus de Carlton-Gardens, en juin 1940, demanda à de Gaulle “au nom de quoi je signe, mon général ?”, et s'entend répondre "au nom de la France, Cassin!”, il regarda autour de lui pour s'assurer qu'aucune personne normalement constituée n'était témoin de la scène. On les aurait pris pour des fous furieux.» (Le Monde, 11 janvier 1996)

12. Maurice Tournier (2002, p. 124) a déjà souligné la proximité qu'il y avait, dans la posture discursive de Jacques Chirac, entre moi et France d'une part, entre Je et Français d'autre part. 
On constate que la formule n'a ni le même poids, ni le même champ d'application symbolique selon les personnes et les époques. Cela signale une dynamique du contenu représentationnel du nom de pays, ouvert à des mises en scènes variées.

\section{Le défigement de la formule}

Dans les discours politiques cités, l'effet d'oracle repose régulièrement sur l'emploi du nom de pays, qui donne une légitimité à la formule. Cependant, le toponyme semble ne plus se suffire à lui-même dans certains contextes, où il se voit accompagné d'expansions destinées à préciser, en la restreignant, sa portée représentationnelle.

L'expansion du nom de pays permet de se positionner, comme le fait Jacques Chirac en accueillant, le 19 septembre 1996, le pape Jean-Paul II «au nom de la France républicaine et laïque». Par cet exercice de langage, tout en rappelant les principes républicains et laïques du groupe qu'il représente, le porte-parole annonce le caractère non religieux de l'échange.

L'acte accompli lui-même permet parfois de doter le toponyme d'une expansion, en vertu des présupposés culturels qu'il véhicule (de cette manière, par exemple, dans la culture chrétienne, «il est généreux de pardonner»). C'est ainsi qu'un ministre français de l'époque est allé pardonner, « au nom de la France généreuse », les massacres de Sétif de 1945 (Libération, 7 mai 1999)...

Mais, dans beaucoup de cas, l'expansion renvoie à une extraction plus qu'à une représentation plus détaillée du groupe représenté; « la France telle et telle » s'oppose en ce sens au groupe représenté par «la France » tout court. On pourrait mentionner ici Céline et Brasillach, qui œuvrent «au nom de la France aux Français» (Le Monde, 15 février 1997); une diplomatie secrète, menée [au Rwanda] "au nom de la France unie, sinon d'une plus grande France» (Libération, 25 septembre 1999); Jean-Pierre Chevènement, qui propose de remettre en mouvement un pays immobile «au nom de la France retrouvée» (7 mars 2002). La formule perd de ce fait en partie son pouvoir de légitimation en devenant programmatique. On a donc affaire à une sorte de défigement sémantique - et non plus lexical ou morphosyntaxique -, qui remet en cause l'unité de l'objet social que le nom de pays cimente grâce à son rôle d'intégrateur sémantique.

Le pas vers le clivage est dès lors facilement franchissable par le biais des expansions. À commencer par l'expression France d'en haut/France d'en bas, dont Sylvianne Rémi-Giraud (2005) montre la capacité de dénonciation, mais aussi de développement de valeurs axiologiques non prévisibles à partir du couple haut/bas pris abstraitement. En effet, en renvoyant constamment au 
monde politique et à l'actualité, les discours qui appuient et qui creusent le clivage retravaillent l'expression et donc, corollairement, la valeur du toponyme. D'autres oppositions, plus ou moins stabilisées au niveau de la forme, émergent au fil de l'actualité et des débats. Ainsi, par exemple, la crise du CPE de début 2006 institue un clivage dans lequel le Premier ministre ${ }^{13}$ et les patrons $^{14}$ se font les porte-paroles de la «France silencieuse», c'est-à-dire celle qui ne manifeste pas dans la rue contre le CPE. Le défigement semble ainsi aller au-delà de l'effet expressif et de la remotivation des termes (voir Fiala et Habert, 1989), en visant une renégociation du contenu symbolique du toponyme.

\section{L’alchimie de la représentation, ou Je suis la France}

De l'effet d'oracle à l'expression même de l'alchimie de la représentation, n'y a-t-il donc qu'un pas? Peut-être, mais il semble difficile à franchir dans le monde politique. L'identification avec la France (je suis la France ${ }^{15}$ ) n'est pas courante. à part le célèbre je suis la France gaullien, énoncé lors de l'arrivée à Londres du général en 1940 (d'après www.diplomatie.gouv.fr), nous avons pu relever uniquement deux $\operatorname{cas}^{16}$ : Jean-Pierre Raffarin s'identifie à la « France d'en bas " sans jamais le formuler directement; Dominique de Villepin est le seul, semble-t-il, à l'avoir dit verbatim, mais au passé: "J'ai compris que j'étais la France. ॥ $^{17}$ Cependant, même dans ces deux cas, l'identification s'appuie sur des représentations symboliques différentes, dues aux valeurs référentielles particulières de chacune des occurrences du nom de pays. Dans le premier cas, on a affaire à une stratégie d'empathisation interdiscursive, via la pluralité des microdiscours véhiculés par l'expression France d'en haut/France d'en bas (voir Rémi-Giraud, 2005, p. 95). Le toponyme renvoie ici au peuple. Dans le second cas, il s'agit d'une identification symbolique

13. «Dès 15 heures à la tribune de l’Assemblée nationale, il [Dominique de Villepin] se sent légitimé pour gouverner au nom de la France silencieuse: “évidemment, j’écoute ceux qui manifestent mais j'écoute aussi ceux qui ne manifestent pas”.» (Le Monde, 9 février 2006)

14. "Sous la signature de Dominique Roy, cette "Lettre des PME de Franche-Comté » fustige ceux qui prennent prétexte du CPE, les “syndicats étudiants nostalgiques d’un printemps idéalisé”, "syndicats de salariés en mal de légitimité” ou “gamins manipulés”. L’organisation patronale des dirigeants de TPE et PME souhaite réagir « au nom de la France qui travaille, qui cotise, qui se tait et se bat pour conserver l'activité donc l'emploi durable." (Le Progrès, 18 mars 2006)

15. Notons l'ambigüité de cette formule en français: s'agit-il d'être ou de suivre? Nous n'avons bien entendu retenu ici que les occurrences relevant de l'identification.

16. Il s'agit de personnes ayant déjà utilisé la formule au nom de la France dans leur discours.

17. «Lorsqu'il est arrivé au Quai d’Orsay, il rapportait qu'il s'était senti fort démuni pour savoir quelle contenance adopter lors d'un premier sommet avec ses homologues. “J'ai compris que j'étais la France”, confiait-il alors. Pour le parodier aujourd'hui, on pourrait dire qu'il s'identifie aux Français. Non pas pour être "comme eux" - même s'il prend le train des vacances avec un banal sac de sport, le concept de France d'en bas lui a toujours semblé idiot et dangereux-, mais "avec eux". » (Le Point, 18 août 2005) 
légitimée par le statut de la personne et par le contexte international; c'est à l'institution de l'état que le toponyme renvoie avant tout. On comprend tous les effets, aussi bien positifs que négatifs, que le contenu associé au nom de pays lors de l'alchimie de la représentation peut avoir sur l'image politique des deux hommes.

De manière plus courante (13 occurrences), de simples citoyens utilisent les formules je suis la France ou nous sommes la France, en rattachant éventuellement des expansions au toponyme: la France du sous-sol, de demain, d'en bas, de toutes les couleurs, éternelle, etc. Si le préalable statutaire de porte-parole est absent de ce registre, cet usage n'est pas pour autant à l'écart de toute alchimie représentationnelle. Car, le nom de pays se situant à la charnière entre groupe et porte-parole, les deux pendants sont impliqués dans la construction de la réalité sociale.

Il y a tout d'abord cette exclamation, attribuée à Abdelaziz Eljaouhari, qui circule telle quelle dans les médias étrangers (anglais, espagnols, italiens, tchèques): «Moi, je suis la France! »18. Cet énoncé semble relever du même phénomène social que l'énoncé nous sommes la France figurant dans un des titres du Nouvel Observateur du 17 novembre 2005. Dans ce titre, un des symboles de la France est évoqué: "Ils veulent une Marianne issue de l'immigration - "Nous sommes la France!" [...]». La première partie du titre est de fait une interprétation de la deuxième. On se rend compte que l'identification avec la France est perçue comme une annonce de revendication, comme un acte non purement référentiel - du fait de l'identification -, mais performatif, du fait des mécanismes représentationnels immédiatement déclenchés par le nom de pays. Ainsi, employer le nom de pays dans ce contexte revient à intervenir dans le champ des représentations symboliques et à prendre des distances par rapport à la déférence sémantique.

D'autres formes d'identification, qui s'emploient notamment à modifier le nom de pays, ont un rôle distinctif d'un point de vue générationnel ou social et marquent souvent un clivage. «Nous sommes la France de demain » est une des formes d'identification les plus revendiquées dans la presse. Souvent attribuée à des lycéens lors des manifestations anti-FN en avril-mai 2002 ou anti-CPE au printemps 2006, elle souligne généralement un écart idéologique intergénérationnel:

Et de refuser la logique économique de cette réforme: «Tout cela répond uniquement à des intérêts financiers. » Avant de se demander: "Si on arrête l'école, que fera-t-on?» Une de ses amies renchérit: «Nous sommes la France de demain.» Une formule que ses camarades de classe reprenaient en chœur dans la foulée. [...] (Le Progrès, 11 février 2005)

18. " "Moi, je suis la France!" cried Abdelaziz Eljaouhari, an activist from another community, decrying Sarkozy's habit of treating him as if he were not. » (The Guardian, 8 juin 2006) 
Mais il peut s'agir aussi, au-delà de l'écart idéologique, d’une réflexion sur la dynamique de la société:

En mai 1968, c'était la fin de la colonisation, il y avait peu de Noirs. La France qui nous dirige a cet âge-là. Moi, je suis une meuf concrète. Les vieux vont mourir. Nous sommes la France de demain. Les peuples bougent, se mélangent. (Ouest-France citant les paroles d'une jeune femme «d'origine africaine », 27 avril 2006)

L'identification à la France d'en bas / du sous-sol sert aussi bien à dénoncer une situation...

Plusieurs cris de révolte ont été entendus comme "Nous sommes la France du sous-sol», "On nous traite comme des animaux». Un nouveau rassemblement est prévu samedi à $10 \mathrm{~h}$. (Associated Press, 6 janvier 2004)

...qu'à argumenter sa position idéologique, en justifiant par exemple le fait de voter «non » à la Constitution européenne:

Un «moi, je suis la France d'en bas » lâché à bout d'arguments à une frangine mieux lotie, qui en a presque pleuré. (Libération, 18 mai 2005)

Ces différents angles d'identification pourraient constituer le ciment des valeurs symboliques futures du nom de pays.

En observant les diverses façons dont les locuteurs, mandatés ou non, s’approprient le nom de pays à travers les formules au nom de la France et je suis / nous sommes la France, on suit la trace des représentations symboliques qu'il véhicule et de leur ajustement social. On constate ainsi une instrumentalisation sociale de l'objet linguistique, qui devient un outil de représentation. Or, comme le soulignent Peter Berger et Thomas Luckmann (1996), l'univers symbolique est par définition problématique et donc toujours susceptible d'être remis en question ${ }^{19}$. Sans être nécessairement des signes de remise en cause, les clivages observés ci-dessus sont symptomatiques d'une renégociation portant sur l'outil même de la représentation symbolique, c'est-àdire le nom de pays. Entre légitimation et production idéologique d'une part et clivage représentationnel d'autre part, le toponyme reste bien ancré dans la réalité sociale, qui influe nécessairement sur son bagage sémantique et symbolique. En retour, au gré des contextes, le nom de pays glisse discrètement ses valeurs dans les discours politiques et quotidiens, en dégageant des postures discursives et en esquissant des portraits représentatifs d'une idéologie ou d'une époque. Le toponyme assume ainsi un rôle de baromètre social, qui pointe en finesse la dynamique de la société.

19. «La légitimation de l'ordre institutionnel est également confrontée à la nécessité continue d'une mise à l'écart du chaos. Toute réalité sociale est précaire. Toutes les sociétés sont des constructions en face du chaos. » (Berger, Luckmann, 1996, p. 142) 


\section{Références}

Berger Peter, Luckmann Thomas, 1996 [1966], La construction sociale de la réalité, Paris, Méridiens Klincksieck.

BOURDIEU Pierre, 1982, Ce que parler veut dire: économie des échanges linguistiques, Paris, Fayard.

- 2001 [1984], "La délégation et le fétichisme politique», Langage et pouvoir symbolique, Paris, Fayard, p. 259-279.

CISLARU Georgeta, 2005, Étude sémantique et discursive du nom de pays dans la presse française avec référence à l'anglais, au roumain et au russe, thèse en sciences du langage, Université de la Sorbonne nouvelle Paris 3.

- 2006a, «Nom de pays et autoreprésentation dans le discours des périodiques nationaux français, anglophones, roumanophones et russes», Les Carnets $d u$ Cediscor, $n^{\circ}$ 9, p. 131-144.

- 2006b, «Nom de pays, nom de peuple: quels usages, quelles identités?» Cahiers de sociolinguistique, $\mathrm{n}^{\circ} 11$, p. 41-62.

DURKHEIM Émile, 1958, «L'état», Revue philosophique, n 148, p. 433-437.

FIALA Pierre, HABERT Benoît, 1989, "La langue de bois en éclats: les défigements dans les titres de presse quotidienne française », Mots. Les langages du politique, $\mathrm{n}^{\circ} 21$, p. 83-98.

GoffMAN Erving, 1974 [1967], Les rites d'interaction, Paris, Minuit.

KAUFMANN Laurence, 2006, "Les voies de la déférence. Sur la nature des concepts socio-politiques », Langage et société, $\mathrm{n}^{\circ}$ 117, p. 89-116.

KRIEg-Planque Alice, 2003, "Purification ethnique». Une formule et son histoire, Paris, CNRS.

LECOLLE Michelle, 2001a, « Métonymie dans la presse écrite : entre discours et langue », Travaux neuchâtelois de linguistique, $\mathrm{n}^{\circ}$ 34 / 35, p. 153-170.

- 2001b, «Personnifications et métonymie dans la presse écrite: comment les différencier?", Semen, $n^{\circ} 15 / 2$, p. 97-112.

Moscovicl Serge, 1984, "The phenomenon of social representations», R. Farr et S. Moscovici éd., Social representations, Cambridge, Cambridge University Press / Paris, MSH, p. 3-69.

Paveau Marie-Anne, 2006, Les prédiscours. Sens, mémoire, cognition, Paris, Presses de la Sorbonne nouvelle.

Putnam Hilary, 1990 [1988], Représentation et réalité, Paris, Gallimard.

RÉMI-GIRAUD Sylvianne, 2005, "France d'en haut/France d'en bas: Raffarin tout terrain », Mots. Les langages du politique, $\mathrm{n}^{\circ} 77$, p. 93-105.

SEARLE John R., 1998 [1995], La construction de la réalité sociale, Paris, Gallimard.

Sıв Loт Paul, 1986, «Moi, général de Gaulle, soldat et chef français, j’ai conscience de parler au nom de la France », Cahiers de praxématique, $n^{\circ}$ 6, p. 7-29.

TOURNIER Maurice, 2002, «De France à Je. La traversée des emplois; cooccurrences et connexions », Propos d'étymologie sociale 3, Lyon, ENS Éditions, p. 111-125. 